\title{
DETERMINANTES DEL EXCESO DE DURACIÓN EN LA EDUCACIÓN TERCIARIA EN CHILE
}

\author{
Sebastián Carpentier ${ }^{1}$
}

\begin{abstract}
RESUMEN
Los estudiantes universitarios chilenos incurren en niveles de sobreduración excesivamente grandes, que generan perjuicios monetarios y socioemocionales considerables. Se busca determinar las principales causas de los excesos de duración, de cara a entregar las primeras luces acerca de cómo disminuirlos y mitigar los efectos negativos que provocan. A través de un análisis multivariado, se realizó un estudio censal de los egresados de la educación superior chilena en la última década, para identificar los factores que incidieron en el exceso de duración percibido por los estudiantes. Así, se distinguieron varios patrones al interior de la educación superior chilena que se alinean con la evidencia recopilada al respecto en el mundo. Se evidenció que los incentivos para terminar a tiempo que impone la posibilidad de perder un beneficio de financiamiento, hacen que estudiantes de iguales características demoren menos en cursar sus estudios superiores. Entonces, extender el beneficio de financiamiento por gratuidad a los años de sobreduración generaría un aumento en el exceso de duración percibido por los estudiantes más vulnerables.
\end{abstract}

Conceptos clave: sobreduración, titulación oportuna, deserción, rezago en educación superior, exceso de duración

\section{DETERMINANTS FOR OVER-DURATION IN HIGHER EDUCATION IN CHILE}

\section{ABSTRACT}

Excessive over-duration of studies among undergraduate Chilean students is generating considerable financial and socioemotional damages. The aim of this work is to identify the main causes of excessive duration of studies in order to generate public policy recommendations to reduce the over-duration and mitigate its negative effects. Through a multivariate analysis, a census study of Chilean university graduates during the last decade was carried out to identify the main factors that influence the excess of duration perceived by students. Thus, several patterns were identified in the Chilean case that are aligned with the evidence collected on this subject worldwide. The study reveals that the incentives to finish on time imposed by the possibility of losing a financial benefit makes students with identical characteristics take less time to complete their studies. Therefore, extending the free financial benefit to the years of excess duration would generate an increase in the over-duration among the most vulnerable students.

Key concepts: over- duration, timely graduation, dropout, lagging behind in higher education, excess of duration

1 Fundación Acción Educar, Santiago, Chile. Contacto: scarpentie@fen.uchile.cl 


\section{Introducción}

Según el informe Education at Glance de la OECD (2019), solo un $16 \%$ de los estudiantes que ingresan a la universidad en Chile logran completar sus estudios en el horizonte temporal determinado por la duración teórica del programa. El porcentaje de titulación oportuna ${ }^{2}$ reportado en Chile resulta ser, por lejos, el más bajo de los países de la OECD, en los que el promedio alcanza un 39\%. No obstante, al ampliar el horizonte de titulación oportuna a la duración formal más tres años, el porcentaje observado en Chile asciende a un 54\%, cifra similar a la reportada en países como Austria, Bélgica, Estonia o Suecia (OECD, 2019; Vossensteyn et al., 2015).

Entonces, la conclusión que se deriva de las cifras informadas por la OECD no es que los estudiantes chilenos tengan un porcentaje de completitud de los estudios superiores particularmente bajo, sino que el tiempo que requieren para completarlos es mayor al que se encuentra predeterminado en la malla curricular. En otras palabras, se puede decir que, en Chile, los estudiantes que completan la educación terciaria incurren en un exceso de duración.

La sobreduración o exceso de duración corresponde a la diferencia entre la duración teórica de los programas y el tiempo que transcurre entre que un individuo se matricula en la educación terciaria y obtiene su grado o licenciatura. Un exceso de duración promedio considerablemente más elevado en Chile incita a realizar las siguientes preguntas: ¿qué provoca que un estudiante demore más de lo estipulado en cursar su carrera? ¿Qué nivel de incidencia tienen los antecedentes académicos y las características socioeconómicas de los estudiantes y su entorno? ¿Cuánto influyen los incentivos económicos asociados a terminar el programa más rápidamente?

La problemática planteada ha sido abordada reiterativamente desde una perspectiva financiera, ya que el exceso de duración implica una carga monetaria adicional importante. Primero, se destaca el

2 Se considera que un estudiante se tituló oportunamente si completó la totalidad de las actividades exigidas por la institución donde realizó sus estudios en el tiempo estipulado por la malla curricular. 
gasto adicional que representa el pago de un año más de arancel, a lo cual se debe sumar los costos correspondientes a arriendos de alojamiento, libros, seguros, licencias, etc. Además, se debe tener en consideración el costo de oportunidad asociado a destinar más tiempo a terminar los estudios, mientras que, alternativamente, el estudiante podría estar trabajando y percibiendo un salario acorde con su profesión. El impacto de los incentivos financieros sobre la titulación oportuna ha sido ampliamente estudiado en países como Noruega (Gunnes, Kirkebøen \& Rønning, 2013; Sten-Gahmberg, 2020), Italia (Garibaldi, Giavazzi, Ichino \& Rettore, 2012), Finlandia (Hämäläinen, Koerselman \& Uusitalo, 2017) y Estados Unidos (ScottClayton, 2015).

Por otro lado, Deming, Hastings, Kane y Staiger (2014), y Herzog (2005) dejaron en evidencia la relevancia que tienen los antecedentes de los últimos años de secundaria sobre la duración de la etapa universitaria de los estudiantes, destacándose como principales predictores los resultados académicos y la calidad del establecimiento de egreso de la educación media ${ }^{3}$. Complementariamente, autores como Allen y Robbins (2010) y Vera-Noriega et al. (2012) demostraron también la incidencia de factores socioemocionales sobre la titulación oportuna de estudiantes universitarios.

El exceso de duración no solo genera perjuicios monetarios y emocionales a los estudiantes y sus familias, sino también un efecto dañino para la sociedad en su conjunto. Por ejemplo, la sobreduración pospone la transición de los egresados hacia el mercado laboral, impactando negativamente la proporción de la fuerza laboral calificada y su eventual contribución a la sociedad a través del aporte fiscal, o las externalidades positivas generadas por una mayor calificación. Peor aún, se ha documentado (Bettinger \& Long, 2009; DesJardins, Ahlburg \& McCall, 2002) que los atrasos en la educación terciaria provocados por mayores requerimientos curriculares se asocian a una disminución de la probabilidad de que los estudiantes concluyan correctamente sus estudios.

3 En Chile se conoce como "educación media" a los últimos cuatro años de la etapa escolar, los cuales corresponden a la educación secundaria alta (clasificación ISCED 3 de la UNESCO) en el resto de América Latina. 
Los principales trabajos en la materia realizados en Chile han tenido como principal objetivo la cuantificación de la magnitud del exceso de duración incurrido por los estudiantes de un grupo acotado de instituciones (Carvajal, Gonzalez, Tassara y Álvarez, 2018; Pey, Durán y Jorquera, 2012; Oriol Granado, Mendoza Lira, Covarrubias Apablaza y Molina López, 2017), lo cual entrega luces de la influencia que podrían tener los distintos factores sobre el exceso de duración, pero no permite extrapolar los resultados a un nivel más general del sistema en su conjunto. Por ende, un estudio censal que incorpore la variación simultánea de los potenciales determinantes de la sobreduración, resulta necesario para orientar políticas públicas destinadas a reducirla y mitigar el impacto perjudicial que esta genera sobre los estudiantes, sus familias, las instituciones y la sociedad.

El análisis mencionado se vuelve aún más relevante si consideramos que el debate acerca de la sobreduración en la educación superior chilena (en adelante, "ES") ha tomado cierta preponderancia, producto de la aplicación de la política de gratuidad ${ }^{4}$. En particular, la ley $N^{\circ} 21.091$ establece que los beneficiarios de la gratuidad que excedan la duración nominal de la carrera perderán el beneficio, teniendo que financiar el 50\% del arancel durante el primer año de atraso — las instituciones de educación superior (en adelante, "IES") asumirán el costo de la otra mitad-y el 100\% a partir del segundo. Las implicancias asociadas a la aplicación del artículo 108 han generado incertidumbre, por lo que resulta crítico analizar qué factores influyen en el exceso de duración para comprender quienes serán los más afectados por esta medida y cuáles son los incentivos que impone sobre estudiantes e IES.

Entonces, se busca responder las interrogantes planteadas y entender cuáles fueron los factores que influyeron en el exceso de duración de los estudios terciarios durante la última década en Chile. Se investiga la presencia de correlaciones y relaciones causales entre la sobreduración incurrida por un estudiante y las características de este último, teniendo en consideración sus antecedentes socioeconómicos

4 Para mayor profundidad, revisar los artículos 82 a 115 de la Ley $N^{\circ} 21.091$, publicada el 29 de mayo de 2018. 
y de la enseñanza media, así como la amplia diversidad de propuestas académicas que presentan las IES y la variedad de mecanismos de financiamiento al que puedan haber optado los individuos.

En lo que sigue se presenta un apartado metodológico en el que se define el concepto de "sobreduración", se caracterizan las fuentes de información utilizadas y se presentan los modelos empleados para identificar los determinantes de la sobreduración. Luego, se presenta estadística descriptiva que permite caracterizarla según diversas clasificaciones de los estudiantes e IES. Posteriormente, se exponen los principales resultados derivados de los modelos. Finalmente, se discuten los resultados obtenidos, teniendo en consideración el alcance y las limitaciones de la metodología empleada y evaluando las potenciales recomendaciones de política pública que podrían inferirse.

\section{Propuesta metodológica}

\subsection{Definición de "sobreduración"}

El horizonte de tiempo definido por las IES para cursar los programas de ES se conoce como la duración formal o nominal de una carrera, el cual oscila entre uno y siete años en la oferta académica terciaria en Chile. No obstante, el tiempo que transcurre desde que un alumno se matricula en la ES hasta que consigue titularse en general excede la duración nominal estipulada por la institución donde estudió. Dicho exceso es conocido como "sobreduración" —o exceso de duración$y$, para determinarlo, se utilizan tres datos: fecha de ingreso a la educación terciaria, fecha de egreso del programa y duración nominal de este último.

Se consideró como "duración formal" de cada carrera aquella que es reportada por las IES y que se encuentra medida en semestres (dos semestres equivalen a un año). Además, se consideró que la cantidad de semestres transcurridos entre la fecha de ingreso y la de egreso corresponde a la duración real incurrida por el estudiante. Entonces, la sobreduración corresponde a:

Sobreduración $n_{i}=$ Duración $_{\text {Real }}-$ Duración Formal Carrera $_{i}$ 
El indicador estaría medido en semestres de exceso en completar la carrera. Además, se crea un indicador que permite comparar el exceso de duración entre individuos que cursaron carreras con una duración teórica dispar. La tasa de sobreduración, que corresponde al exceso porcentual de duración respecto de la duración teórica de los programas, se construye de la siguiente manera:

$$
\text { Tasa de Sobreduración } n_{i}=\frac{\text { Sobreduración }_{i}}{\text { Duración Formal Carrera }_{i}}
$$

La tasa obtenida en términos porcentuales permite comparar el exceso de duración incurrido por todos los estudiantes, independiente de que la duración formal de las carreras cursadas varíe de un egresado a otro. La tasa de sobreduración promedio reportada para los 866.495 egresados del pregrado en Chile durante la última década asciende a un $32 \%$, es decir, los estudiantes demoraron, en promedio, un $32 \%$ más de lo que dicta la malla curricular en egresar de sus carreras.

\subsection{Fuentes de información}

El presente estudio busca identificar los determinantes del exceso de duración en la ES. Para ello se debe tener en consideración un set de variables muy diverso, que podrían estar condicionando o afectando los resultados académicos de los estudiantes. A continuación se presentan las ocho bases de datos utilizadas para determinar el exceso de duración incurrido por cada uno de los estudiantes que obtuvo su título profesional en Chile durante la última década, permitiendo además caracterizar a los alumnos y sus trayectorias académicas.

En el portal web Datos Abiertos del Ministerio de Educación (en adelante Mineduc) se encuentran las bases de datos de Matriculados en Educación Superior ${ }^{5}$ y Titulados de Educación Superior ${ }^{6}$. Al

5 Disponible en: http://datos.mineduc.cl/dashboards/20195/descarga-bases-de-datosmatricula-en-educacion-superior/

6 Disponible en: http://datos.mineduc.cl/dashboards/20207/descarga-bases-de-datostitulados-de-educacion-superior/ 
combinar estas dos fuentes se pudo identificar y caracterizar a todos los estudiantes que obtuvieron su título de pregrado durante la última década, según su año de ingreso y de egreso, la institución y carrera que cursaron, etc. Se obtuvo una base de datos con la información de los 866.495 alumnos que comenzaron sus carreras desde el año 2000 en adelante y que culminaron sus estudios de pregrado entre 2010 y 2019.

Luego, con el fin de determinar el origen socioeconómico de los estudiantes y cuál fue el mecanismo de financiamiento empleado para costear sus carreras, se consultaron tres bases de datos. En el mismo portal de Datos Abiertos del Mineduc se encuentran las bases de "Postulaciones"7 y "Asignaciones" 8 de becas y beneficios de financiamiento ofrecidos por el Estado. Además, se dispuso de la base de datos histórica de "Asignaciones del Crédito con Garantía Estatal” (CAE), entregada por la Comisión Ingresa. Entrelazando la información aportada por estas tres fuentes, se identificó el quintil de ingresos y el mecanismo de financiamiento utilizado para costear sus estudios de la mayoría de los egresados de la última década.

La base "Notas de Enseñanza Media y Percentil en Jóvenes" —disponible en el portal de Datos Abiertos del Mineducpermitió caracterizar los antecedentes académicos de la educación media de un $70 \%$ de los egresados de la ES, así como identificar el establecimiento y año de egreso. Además, para considerar una medición de vulnerabilidad multidimensional se incorporó el Índice de Vulnerabilidad Escolar (IVE) de la comuna de la cual egresaron los estudiantes, información que se encuentra disponible en el sitio web de la Junta Nacional de Auxilio Escolar y Becas (JUNAEB) ${ }^{10}$.

Por último, en el portal web Mi Futuro se puede encontrar información sobre empleabilidad e ingreso promedio de los egresados

7 Disponible en: http://datos.mineduc.cl/dashboards/20208/descarga-base-de-datospostulaciones-a-becas-y-creditos-en-educacion-superior/

8 Disponible en: http://datos.mineduc.cl/dashboards/20209/descarga-base-de-datosasignaciones-de-becas-y-creditos-en-educacion-superior/

9 Disponible en: http://datos.mineduc.cl/dashboards/20389/descarga-de-base-de-datos-notasde-ensenanza-media-y-percentil-en-jovenes/

10 Disponible en: https://www.junaeb.cl/ive 
de cada una de las carreras disponibles en la oferta académica. Estos datos permiten determinar el costo de oportunidad que representa un año adicional de estudio.

\subsection{Metodología de análisis multivariado de la sobreduración}

Para identificar los determinantes del exceso de duración se emplean tres especificaciones distintas, en un modelo de regresión lineal múltiple, a través de mínimos cuadrados ordinarios con error estándar robusto. Esto permite cuantificar la variación percibida por la variable de estudio, el exceso de duración percibido por un estudiante ante una variación marginal de las variables explicativas del modelo, manteniendo todo lo demás constante. Así, al comparar a individuos de características observables similares se busca determinar el impacto que tienen, en promedio, cada una de las variables explicativas sobre el exceso de duración. La especificación del primer modelo econométrico empleado es la siguiente:

$$
\begin{aligned}
T_{S D}= & \beta_{0}+M_{i}^{*} \beta_{1}+\text { Edad }_{i}^{*} \beta_{2}+V_{i}^{*} \beta_{3}+\text { Decil }_{\text {NEMi }} * \beta_{4}+\text { Municipal }_{i} * \beta_{5}+ \\
& \text { Subv }_{i}^{*} \beta_{6}+\text { Código }_{\text {IES }} * \varnothing+\text { Area }_{\text {SIES }} * \alpha+\text { Región }^{*} \gamma+\varepsilon_{i}
\end{aligned}
$$

La variable dependiente $T S D_{i}$ corresponde a la tasa de sobreduración (en adelante, "TSD") incurrida por el individuo. La variable binaria $M_{i}$ toma el valor 1 si el estudiante es mujer y 0 si es hombre, mientras que Edad corresponde a la edad del alumno al ingresar a la carrera. La variable $V_{i}$ señala si el alumno era vulnerable ${ }^{11}$ al comenzar sus estudios superiores: es igual a 1 si pertenecía al 60\% más pobre de la población.

Luego, se incorporaron las variables que describían los antecedentes de la educación media del individuo. Decil ${ }_{N E M i}$ indica el decil de notas de egreso de la enseñanza media del estudiante, el cual es más elevado mientras mejores hayan sido sus notas en comparación a la generación con la cual egresó del establecimiento. Las variables Municipal y Subv $_{i}$ son variables binarias que identifican la

11 Se construyó un indicador de "vulnerabilidad" basado en el mismo umbral de corte que el beneficio de financiamiento por gratuidad: se considera que el individuo es vulnerable si pertenece a alguno de los tres primeros quintiles de ingreso. 
dependencia del establecimiento de educación media del estudiante. La primera es igual 1 si el individuo egresó de un colegio municipal y 0 si es que no fue así. De igual manera, Subv toma el valor 1 si el estudiante proviene de un colegio subvencionado y 0 si es que proviene de otro tipo de establecimiento. En el caso de que ambas sean iguales a 0 se infiere que el estudiante egresó de un colegio particular pagado.

Por otro lado, el set de variables Código ${ }_{I E S}$ está constituido por 181 variables binarias que determinan la IES en la cual el alumno cursó sus estudios superiores. Análogamente, Área $a_{\text {SIES }}$ es un set de 250 variables binarias que determinan el área genérica de la carrera que cursó el estudiante. La categorización de área genérica realizada por el Mineduc ${ }^{12}$ agrupa carreras que tienen nombres y estructuras curriculares similares. Por último, Región incluye 15 variables binarias que determinan la región donde el individuo estudió. Los coeficientes obtenidos para estos tres grupos de variables de control no se presentan en los resultados, ya que fueron incluidos únicamente con el fin de controlar por las diferencias observadas. El término de "error" $\varepsilon_{i}$ captura todas las variaciones de la TSD que no se explican por la variación de las variables dependientes del modelo.

Posteriormente se emplearon dos especificaciones adicionales para complementar los resultados derivados del primer modelo. En el modelo $N^{\circ} 2$ se añaden cinco variables que permiten identificar los beneficios de financiamiento que obtuvo el estudiante para costear sus estudios y los costos de oportunidad asociados con estudiar un año más. La variable binaria Crédito es igual a 1 si tomó algún crédito y 0 si no, Beca $_{i}$ toma el valor 1 si obtuvo alguna beca y 0 si no fue así, mientras que Gratuidad ${ }_{i}$ equivale a 1 si el individuo fue beneficiario de la gratuidad durante su paso por la ES. Por otra parte, $\ln Y_{i}$ y $\ln A_{i}$ corresponden al logaritmo del ingreso esperado al egresar y al logaritmo del arancel anual de la carrera. El primero permite evaluar la variación de la sobreduración ante aumentos porcentuales

12 El Servicio de Información de Educación Superior (SIES), del Ministerio de Educación de Chile, es la entidad encargada de desarrollar y mantener un Sistema Nacional de Información de la Educación Superior, que contenga los antecedentes necesarios para la adecuada aplicación de las políticas públicas destinadas al sector. 
del ingreso esperado al egresar, mientras que el segundo explicaría los cambios reportados en el exceso de duración en presencia de un arancel más elevado.

Por último, para incorporar un indicador de vulnerabilidad multidimensional se aplica una tercera especificación econométrica, en la cual se añade el Índice de Vulnerabilidad Escolar (IVE) de la comuna en la cual el estudiante cursó su enseñanza media. Este índice de vulnerabilidad escolar es construido por la JUNAEB y se basa en la interacción de una multiplicidad de factores que se asocian a un mayor o menor riesgo social, económico, psicológico, cultural y/o ambiental, riesgos que podrían producir una desventaja comparativa entre sujetos, familias o comunidades. El valor del IVE varía entre 0 y 1 , en el que un valor más alto se asocia con una mayor vulnerabilidad escolar.

Típicamente, las IES son categorizadas en tres grupos: los centros de formación técnica (en adelante, "CFT"), los institutos profesionales (en adelante, "IP") y las universidades. Producto de las diferencias reportadas entre los tres grupos de IES en la duración nominal de las carreras, en la TSD promedio y en los indicadores de éxito académico, se optó por estimar cada modelo de regresión por separado para cada tipo de IES. Por ende, se realizaron en total nueve modelos de regresión distintos: tres para cada tipo de IES. Para resumir las variables explicativas incorporadas en los modelos se elaboró la tabla $\mathrm{N}^{\circ} 1$, en la cual se puede apreciar el valor promedio, el mínimo, el máximo y el número de observaciones disponibles para cada variable dependiente mencionada: 
Tabla ${ }^{\circ} 1$

Caracterización de las variables explicativas del modelo

\begin{tabular}{lcccc}
\hline Variable & Promedio & Mínimo & Máximo & Observaciones \\
\hline Mujer (Binaria) & 0,601 & 0 & 1 & 866.495 \\
Edad al ingresar & 22,147 & 15 & 59 & 866.495 \\
Vulnerable (Binaria) & 0,524 & 0 & 1 & 866.495 \\
Decil de NEM & 6,387 & 0 & 10 & 600.752 \\
Municipal (Binaria) & 0,397 & 0 & 1 & 600.752 \\
Subvencionado (Binaria) & 0,486 & 0 & 1 & 600.752 \\
Crédito (Binaria) & 0,418 & 0 & 1 & 866.495 \\
Beca (Binaria) & 0,276 & 0 & 1 & 866.495 \\
Gratuidad (Binaria) & 0,049 & 0 & 1 & 866.495 \\
Logaritmo del Ingreso Esperado & 13,579 & 12,77 & 14,85 & 866.441 \\
Logaritmo del Arancel & 14,291 & 11,25 & 15,71 & 851.692 \\
Índice de Vulnerabilidad Escolar & 0,618 & 0,322 & 1,000 & 568.749 \\
\hline
\end{tabular}

Fuente: elaboración propia basada en datos del Mineduc.

\section{Estadística descriptiva}

A continuación se presenta una estadística descriptiva diversa, en la que se evidencian las diferencias de sobreduración reportadas en los distintos segmentos de la población de estudio. Dentro de las variables de interés particular se destacan el origen socioeconómico, el género, la dependencia del establecimiento de educación media de origen, el mecanismo de financiamiento, entre otras.

\subsection{Origen socioeconómico}

El cruce de la base de datos de los titulados de la ES con la información reportada por los mismos estudiantes a través del FUAS ${ }^{13}$, permitió identificar el quintil de ingresos al que pertenecía un $67 \%$ de la población estudiada al momento de ingresar a la ES. No fue posible caracterizar socioeconómicamente al 33\% restante, ya que jamás postularon a algún beneficio de financiamiento estatal, lo cual sugiere que pertenecen a los sectores más favorecidos, porque estaban en

13 El Formulario Único de Acreditación Socioeconómica (FUAS) es el documento de postulación a becas, créditos y gratuidad, mediante el cual se determina el decil de ingresos al que pertenece cada postulante. Por tanto, todo estudiante que haya postulado a algún beneficio de financiamiento estatal, independiente de si logró ser beneficiario o no, posee una caracterización socioeconómica. 
condiciones de financiar sus estudios por sus propios medios, sin solicitar ningún tipo de ayuda financiera. Como se puede apreciar en la tabla $\mathrm{N}^{\circ} 2$, en la que se expone la cantidad de titulados por quintil de ingresos y el promedio de sobreduración de cada grupo, el número de titulados que se logró identificar en los quintiles 4 y 5 es notablemente menor al de los tres primeros quintiles, lo cual respalda la hipótesis de que los individuos "sin información" pertenecían a los quintiles de mayores ingresos ${ }^{14}$.

Tabla $\mathrm{N}^{\circ} 2$

Sobreduración promedio según quintil de ingresos

\begin{tabular}{lcccc}
\hline Quintil de ingreso & Titulados & \% de titulados & Sobreduración & TSD \\
\hline Quintil 1 y 215 & 341.246 & $39,4 \%$ & 1,99 & $30,9 \%$ \\
Quintil 3 & 112.803 & $13,0 \%$ & 2,03 & $29,7 \%$ \\
Quintil 4 & 84.904 & $9,8 \%$ & 2,25 & $29,9 \%$ \\
Quintil 5 & 41.775 & $4,8 \%$ & 2,09 & $28,5 \%$ \\
Sin información & 285.767 & $33,0 \%$ & 2,97 & $36,0 \%$ \\
TOTAL & 866.495 & $100 \%$ & 2,35 & $32,2 \%$ \\
\hline
\end{tabular}

Fuente: elaboración propia basada en datos del Mineduc y la Comisión Ingresa.

Las cifras presentadas sugieren, en primera instancia, que no existe una diferencia relevante en el exceso de duración incurrido por los estudiantes provenientes de los distintos sectores socioeconómicos. La sobreduración promedio medida en semestres se aproxima a 2 en casi todos los quintiles, lo que equivaldría a un año de retraso respecto de la duración formal de las carreras. Al analizar la TSD se constata una tendencia similar, ya que se sitúan todas en torno al 30\%.

Sin embargo, a causa de que los quintiles 4 y 5 están subrepresentados por ausencia de información, es esperable que las cifras presentadas en la tabla $N^{\circ} 2$ para dichos grupos estén subestimadas. Para corregir dicho efecto y visualizar de forma más real la correlación entre el nivel de ingresos y el exceso de duración,

14 Este es un supuesto utilizado regularmente por el Mineduc para caracterizar el origen socioeconómico de los matriculados en la educación superior.

15 Las bases de datos de postulaciones a través del FUAS y asignaciones de becas, agrupan a los dos primeros quintiles de ingreso en una misma categoría llamada "Quintil 1 y 2", razón por la cual, a lo largo del estudio, los individuos provenientes de dichos quintiles son agrupados en una misma categoría. 
se aplicará el supuesto que utiliza el Mineduc para determinar la distribución socioeconómica de la matrícula de ES: si un estudiante no solicitó nunca una ayuda estatal, se asume que proviene de una familia perteneciente al $40 \%$ más rico del país.

Luego se construyó un indicador de "vulnerabilidad", basado en el mismo umbral de corte que hoy tiene el beneficio de financiamiento por gratuidad. De esta forma, se consideró que un individuo era vulnerable si pertenecía al $60 \%$ más pobre. Por el contrario, si provenía del quintil 4 o 5 - o bien jamás solicitó algún beneficio de financiamiento estatal — se asumió que no era vulnerable. Así, se obtuvo que un 52,4\% de los estudiantes que obtuvieron un título en la última década provenían de hogares vulnerables.

Tabla $\mathrm{N}^{\circ} 3$.

Sobreduración promedio según vulnerabilidad al ingreso

\begin{tabular}{lccc}
\hline Indicador de vulnerabilidad & Titulados & Sobreduración & TSD \\
\hline Vulnerable al ingresar & 454.049 & 2,00 & $30,1 \%$ \\
No vulnerable al ingresar & 412.446 & 2,74 & $34,0 \%$ \\
TOTAL & 866.495 & 2,35 & $32,2 \%$ \\
\hline
\end{tabular}

Fuente: elaboración propia basada en datos del Mineduc y la Comisión Ingresa.

En la tabla $\mathrm{N}^{\circ} 3$ se observa que la sobreduración promedio de los estudiantes provenientes de sectores vulnerables es más baja. En términos relativos, la TSD observada en los estudiantes no vulnerables es un 13\% más elevada en promedio que aquella reportada para el grupo de individuos vulnerables. La relación observada no implica necesariamente que exista un vínculo causal, ya que la mayor TSD observada en los estudiantes provenientes de sectores más favorecidos podría explicarse por diversos factores que sean más recurrentes en dicho grupo.

\subsection{Antecedentes de la enseñanza media}

Se ha documentado en diversos estudios que la dependencia de origen de los establecimientos de educación media correlaciona considerablemente con el quintil de ingresos predominante entre sus alumnos. Esto es, la proporción de alumnos provenientes de los quintiles de ingresos más desfavorecidos es mucho más elevada 
en los establecimientos municipales, mientras que en los colegios particulares pagados predomina la presencia de alumnos de hogares más favorecidos (Valenzuela, Allende González, Gómez Vera y Trivelli, 2015). Para ilustrar lo anterior se construyó la tabla $\mathrm{N}^{\circ} 4$, en la cual se puede apreciar la proporción de alumnos vulnerables según la dependencia de origen del establecimiento de educación media y la sobreduración promedio de los egresados de cada tipo de dependencia:

Tabla $\mathrm{N}^{\circ} 4$

Sobreduración promedio observada según dependencia de origen

\begin{tabular}{lccc}
\hline Dependencia de origen & Titulados & \% de alumnos vulnerables & TSD \\
\hline Municipal & 238.647 & $71,3 \%$ & $31,6 \%$ \\
Subvencionado & 291.829 & $60,5 \%$ & $29,8 \%$ \\
Particular pagado & 70.276 & $10,5 \%$ & $24,7 \%$ \\
TOTAL & 600.752 & $58,9 \%$ & $29,9 \%$ \\
\hline
\end{tabular}

Fuente: elaboración propia basada en datos del Mineduc y la Comisión Ingresa.

Por un lado, en la tabla $\mathrm{N}^{\circ} 3$ se observa una TSD promedio menor en los individuos provenientes de hogares más vulnerables. Por el contrario, podemos notar que los egresados de colegios particulares incurrieron en un exceso de duración promedio considerablemente más bajo que sus pares, a pesar de que su concentración de alumnos vulnerables se reduce tan solo a un $10 \%$. Paralelamente, un $71 \%$ de los egresados de colegios municipales provenía de hogares vulnerables, mientras en este tipo de establecimientos se observa un mayor promedio en la TSD. A priori, pareciera que la dependencia del establecimiento tiene mayor influencia en el exceso de duración en el que incurrirá el estudiante en su paso por la ES que la vulnerabilidad de su hogar. No obstante, la hipótesis anterior debiera ser respaldada por un análisis multivariado para evaluar la existencia de un vínculo causal.

Luego, resulta interesante analizar si el promedio de notas de la enseñanza media (NEM) correlaciona con la sobreduración incurrida por el estudiante en la ES. Parece lógico que un individuo que tuvo un buen rendimiento académico y que, por consiguiente, ingresa a la ES con bases de conocimientos y habilidades más sólidas, tendrá 
más herramientas para cumplir con su malla curricular en el tiempo estipulado. Esto ha sido ampliamente documentado en la literatura internacional (Allen, \& Robbins, 2010; Deming et al., 2014) y los resultados expuestos en la tabla $\mathrm{N}^{\circ} 5$ sugieren una conclusión similar para Chile:

Tabla No 5

Sobreduración según el decil de notas de enseñanza media

\begin{tabular}{lcc}
\hline Decil de NEM & Titulados & TSD \\
\hline Decil 1 & 24.535 & $36,1 \%$ \\
Decil 2 & 40.941 & $34,3 \%$ \\
Decil 3 & 47.377 & $32,7 \%$ \\
Decil 4 & 53.336 & $32,1 \%$ \\
Decil 5 & 59.224 & $31,5 \%$ \\
Decil 6 & 61.901 & $30,6 \%$ \\
Decil 7 & 67.679 & $30,1 \%$ \\
Decil 8 & 72.968 & $28,9 \%$ \\
Decil 9 & 78.024 & $27,7 \%$ \\
Decil 10 & 94.767 & $24,8 \%$ \\
\hline TOTAL & 600.752 & $29,9 \%$ \\
\hline
\end{tabular}

Fuente: elaboración propia basada en datos del Mineduc y la Comisión Ingresa.

Para dilucidar la existencia de una correlación positiva entre el rendimiento académico y el exceso de duración, se clasificó a los titulados según su decil de notas de egreso de la enseñanza media. Entonces, un individuo perteneciente al primer decil corresponde al $10 \%$ de las peores notas de su generación de egreso, mientras que uno perteneciente al decil 10 habría estado dentro del 10\% de mejores calificaciones. Se observa que los estudiantes que se graduaron de la educación media con una mejor posición relativa percibieron niveles de sobreduración menores.

\subsection{Tipo de institución y área de estudios}

Utilizando la clasificación institucional del SIES se construyó la tabla $N^{\circ} 6$, en la que se exhibe el exceso de duración y la vulnerabilidad observada en cada tipo de institución de ES: 
Tabla $\mathrm{N}^{\circ} 6$

Exceso de duración promedio por tipo de institución

\begin{tabular}{lccc}
\hline Tipo de IES & \% de titulados & $\begin{array}{c}\text { \% de alumnos } \\
\text { vulnerables }\end{array}$ & TSD \\
\hline Centros de formación técnica & $19,6 \%$ & $67,2 \%$ & $41,0 \%$ \\
Institutos profesionales & $29,5 \%$ & $66,1 \%$ & $27,6 \%$ \\
Universidades estatales CRUCH & $13,7 \%$ & $32,7 \%$ & $33,9 \%$ \\
Universidades privadas CRUCH & $11,5 \%$ & $34,7 \%$ & $34,3 \%$ \\
Universidades privadas no CRUCH & $25,7 \%$ & $43,7 \%$ & $28,8 \%$ \\
TOTAL & $100 \%$ & $52,4 \%$ & $32,2 \%$ \\
\hline
\end{tabular}

Fuente: elaboración propia basada en datos del Mineduc y la Comisión Ingresa.

De la estadística presentada se infiere que la proporción de vulnerabilidad dentro de las instituciones está lejos de ser el factor más determinante del exceso de duración en el que incurren sus alumnos. Los CFT presentan la sobreduración promedio más alta, a pesar de que dos tercios de sus titulados provengan de hogares vulnerables, mientras que los IP reportan el exceso de duración promedio más bajo, aun cuando se observa un nivel de vulnerabilidad casi idéntico al de los CFT.

Por otro lado, se ha demostrado que la elección del programa y el área de estudios por parte de los estudiantes afecta el exceso de duración en el que incurrirán al cursar sus estudios superiores. En particular, se argumenta que, en áreas en las que los requerimientos curriculares son más específicos y numerosos, se genera una extensión más pronunciada del paso por el pregrado (Adelman, 2006). Adicionalmente, se demostró que en las áreas de estudio en las que los titulados perciben mayores ingresos al egresar, el exceso de duración tiende a ser menor (DesJardins et al., 2002). Para tener una primera aproximación a la noción expuesta se elaboró la tabla $N^{\circ} 7$, en la cual se presentan los promedios de sobreduración de los egresados de cada área del conocimiento según la clasificación elaborada por la OECD. 
Tabla $\mathrm{N}^{\circ} 7$

Sobreduración por área del conocimiento

\begin{tabular}{lccc}
\hline Área del conocimiento & \% de titulados & $\begin{array}{c}\text { \% de alumnos } \\
\text { vulnerables }\end{array}$ & TSD \\
\hline Administración y Comercio & $16,7 \%$ & $49,1 \%$ & $31,4 \%$ \\
Agropecuaria & $2,3 \%$ & $47,0 \%$ & $48,2 \%$ \\
Arte y Arquitectura & $4,7 \%$ & $37,9 \%$ & $34,9 \%$ \\
Ciencias Básicas & $1,2 \%$ & $35,0 \%$ & $47,8 \%$ \\
Ciencias Sociales & $9,3 \%$ & $47,6 \%$ & $23,4 \%$ \\
Derecho & $2,8 \%$ & $34,7 \%$ & $49,5 \%$ \\
Educación & $17,0 \%$ & $58,5 \%$ & $25,1 \%$ \\
Humanidades & $0,8 \%$ & $35,4 \%$ & $31,3 \%$ \\
Salud & $24,0 \%$ & $59,3 \%$ & $28,1 \%$ \\
Tecnología & $21,2 \%$ & $52,3 \%$ & $41,5 \%$ \\
TOTAL & $100 \%$ & $52,4 \%$ & $32,2 \%$ \\
\hline
\end{tabular}

Fuente: elaboración propia basada en datos del Mineduc y la Comisión Ingresa.

Al analizar los niveles de sobreduración de los egresados de las distintas áreas del conocimiento, se obtiene una heterogeneidad sorprendente. En un lado se aprecian áreas con un número importante de titulados, como Ciencias Sociales, Educación o Salud, en las que la sobreduración promedio ronda el 25\%. Contrariamente, en áreas más específicas, como Derecho, Ciencias Básicas o el sector Agropecuario, el exceso de duración promedio roza el 50\%.

También resulta sorpresiva la tendencia obtenida al estudiar la variación conjunta de la proporción de vulnerabilidad de cada área y la sobreduración promedio de sus estudiantes. De hecho, al estimar el coeficiente de correlación entre ambas se obtiene -0,534, lo cual deja en evidencia una relación negativa de magnitud relevante. A saber, áreas con una proporción de alumnos vulnerables un 10\% más alta presentan, en promedio, una sobreduración un 5\% más baja.

La obtención de resultados tan diametralmente distintos por área podría estar asociada a los incentivos monetarios de los estudiantes y al nivel de sobrecarga académica impuesto por las IES. Si bien no se cuenta con información que permita cuantificar la exigencia académica, sí se puede determinar el salario que podría esperar recibir un estudiante al culminar sus estudios. Además, conocer el mecanismo mediante el cual los individuos financiaron 
sus programas permite describir las presiones financieras percibidas por los estudiantes y los costos de oportunidad asociados a los años adicionales destinados a terminar sus carreras, en lugar de recibir ingresos laborales.

\subsection{Mecanismo de financiamiento}

Gracias a la información publicada por el Mineduc se pudo identificar el mecanismo de financiamiento utilizado por los estudiantes para costear sus estudios. Para aquellos individuos que jamás obtuvieron un beneficio financiero se asumió que costearon el valor de sus programas con recursos privados. Los egresados pueden haber optado a tres tipos de beneficios distintos: créditos, becas y gratuidad.

El primer grupo corresponde a los dos créditos de educación superior financiados o subsidiados por el Estado (CAE o FSCU). En segundo lugar se agrupó en la categoría "becas" a todos los estudiantes que hayan obtenido alguna de las becas de arancel ofrecidas y entregadas por el Ministerio de Educación. Por último, se consideró a la gratuidad como una última posibilidad de beneficio financiero. Un mismo individuo puede haber optado a uno o a varios de los beneficios posibles, llegando incluso, en algunos casos, a recibir los tres tipos de ayudas estatales.

De la tabla $\mathrm{N}^{\circ} 8$ se desprende que un $56,4 \%$ de los titulados de pregrado en la última década fue beneficiario de al menos una de las ayudas financieras entregadas por el Estado. Luego, se destaca que quienes no gozaron de ningún beneficio, en general tienen un exceso de duración más elevado: cinco puntos porcentuales más de sobreduración en promedio. El resultado anterior se alinea con la evidencia encontrada por Garibaldi et al. (2012), de que los estudiantes que asumen que tendrán que pagar más en caso de atrasarse en completar sus estudios tienden a esforzarse más en terminarlos a tiempo. 
Tabla No8

Sobreduración según tipo de beneficio financiero recibido

\begin{tabular}{llccc}
\hline Tipo de beneficio & & Titulados & $\%$ de titulados & TSD \\
\hline 1 beneficio & Solo crédito & 237.065 & $27,4 \%$ & $33,0 \%$ \\
& Solo beca & 103.347 & $11,9 \%$ & $26,5 \%$ \\
\multirow{2}{*}{2 beneficios } & Solo gratuidad & 5.689 & $0,7 \%$ & $23,0 \%$ \\
& Crédito y beca & 106.360 & $12,3 \%$ & $29,7 \%$ \\
& Crédito y gratuidad & 6.829 & $0,8 \%$ & $22,3 \%$ \\
3 beneficios & Beca y gratuidad & 17.728 & $2,0 \%$ & $17,8 \%$ \\
Ningún beneficio & & 12.099 & $1,4 \%$ & $24,7 \%$ \\
TOTAL & & 377.378 & $43,6 \%$ & $35,1 \%$ \\
\hline
\end{tabular}

Fuente: elaboración propia basada en datos del Mineduc y la Comisión Ingresa.

En efecto, quienes son beneficiarios de la gratuidad no desembolsan ni un peso de su bolsillo para pagar el arancel de su carrera, situación que cambia instantáneamente en caso de exceder la duración formal de esta última, ya que tendrían que pagar el 50\% del arancel durante el primer año de exceso y el 100\% del segundo en adelante. Si hablamos de los becados la situación es similar, ya que, en caso de atrasarse, arriesgan la pérdida del beneficio y tendrían que costear ellos lo que financia la beca. En el caso de los créditos también existe perjuicio en caso de atraso: financiar un año más de carrera pidiendo crédito no solo genera un aumento del monto adeudado, sino que también implica el pago de un año adicional de intereses.

Entonces, no solamente queda evidenciado que los individuos que tendrán que pagar más si se atrasan se titulan antes en promedio, sino que, además, el efecto constatado se acentúa en la medida en que el incentivo financiero a culminar a tiempo es relativamente mayor. Como se explicó recientemente, quienes gozan de gratuidad son quienes percibirían un perjuicio financiero más grande si se atrasan y son precisamente ellos quienes tienen una sobreduración promedio más baja (del orden del 20\%). Los siguen los becados, con un exceso de duración promedio del $27 \%$, y luego quienes estudian con algún crédito, cuya sobreduración media asciende a un 31,5\%. 


\subsection{Costo de oportunidad}

Por otro lado, se busca dilucidar si existe una correlación negativa entre el costo de oportunidad monetario que implica estudiar por un año más y la TSD. Se intuye que, si el costo que un individuo debe asumir por atrasarse es más elevado, entonces se esforzará más en completar sus estudios a tiempo y así evitar percibir dicho costo. El coste de oportunidad asociado al atraso no incluye únicamente el valor del arancel, sino también el ingreso que se podría percibir en caso de egresar a tiempo. Por ende, se explora la presencia de una correlación entre la sobreduración, el arancel de la carrera y el ingreso que percibe un egresado promedio.

Al estimar la correlación entre el arancel pagado y el exceso de duración se obtiene un coeficiente negativo de magnitud pequeña $(-0,0593)$, lo cual sugiere que, a medida que aumenta el arancel, se observan leves diminuciones en la sobreduración promedio. Lo mismo sucede en el caso del ingreso esperado al egresar, en el que se obtiene un coeficiente de correlación de -0,0218 con la TSD.

\section{Resultados de los modelos de regresión}

Al observar los resultados de los modelos de regresión expuestos en la tabla $N^{\circ} 9$, podemos percatarnos de que todas las variables explicativas incluidas en el modelo 1 son estadísticamente significativas, con un nivel de confianza del 99\%. Lo mismo ocurre con los tres modelos estimados para el sector universitario. Es decir, todas las variables incluidas se relacionan de manera significativa con la sobreduración incurrida por los estudiantes, ayudando a predecir y a explicar el valor de esta última. Al analizar los coeficientes obtenidos, se dimensiona la magnitud de la relación existente entre cada una de las variables y el exceso de duración en el pregrado. 


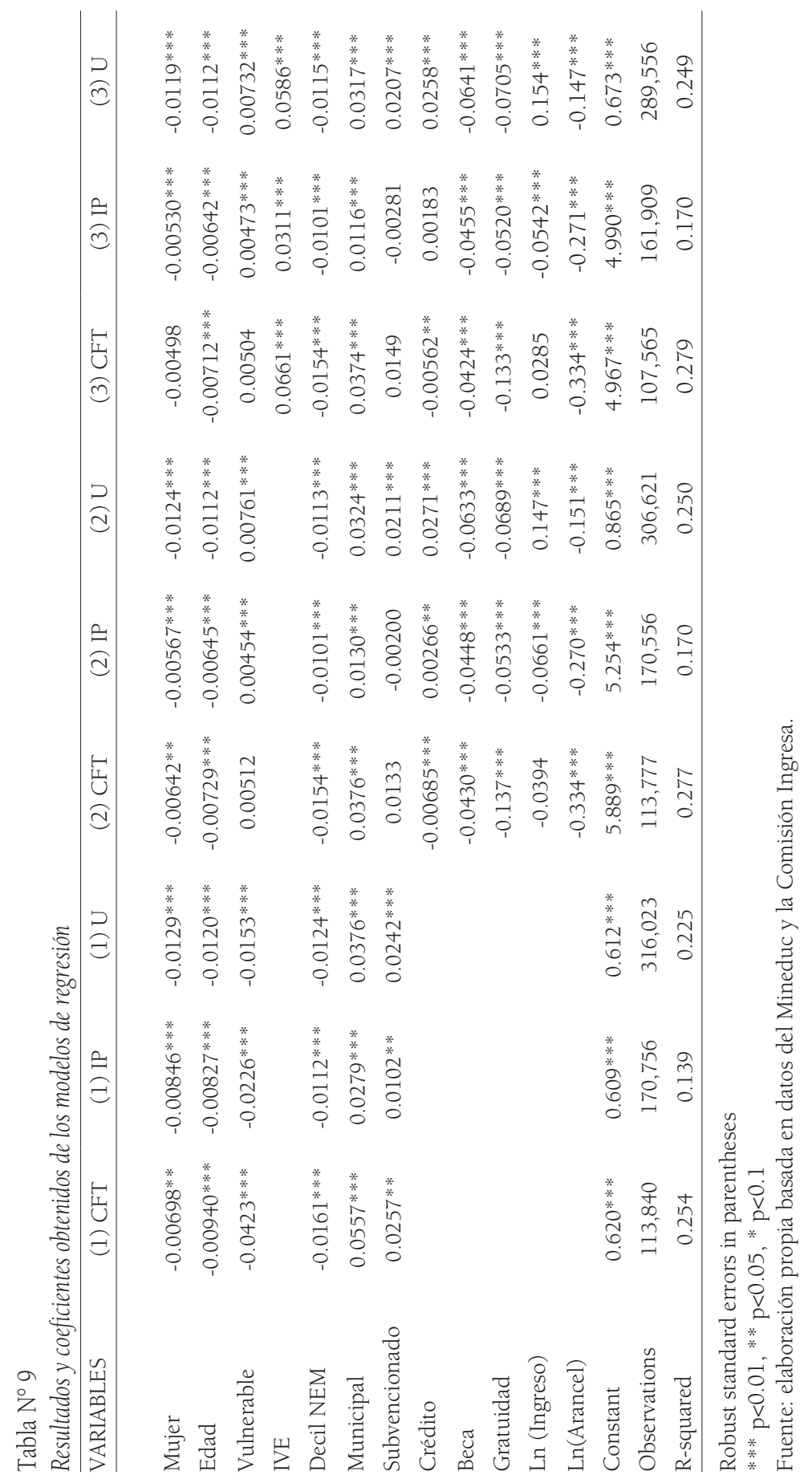




\subsection{Brecha de género}

Primero, se observa que las mujeres, de iguales características observables que sus compañeros del género opuesto, incurrirán en una sobreduración menor. Este efecto es particularmente relevante en las carreras universitarias, en las cuales las mujeres perciben una TSD 1,2 puntos porcentuales más baja que sus pares que estudiaron las mismas carreras y que presentaron una trayectoria académica similar. Esto es equivalente a afirmar que los hombres que asisten a la universidad incurren en un exceso de duración una semana más grande ${ }^{16}$ que las mujeres, ceteris paribus.

El coeficiente obtenido está alineado con la evidencia encontrada por Carvajal et al. (2018) al estudiar a los egresados de carreras profesionales de la Universidad de Playa Ancha, quienes rechazaron la hipótesis de que no existen diferencias significativas entre los efectos del género como predictor de la sobreduración. Los resultados obtenidos en el presente estudio permiten extrapolar los resultados vislumbrados para una casa de estudios a todo el sistema de ES de Chile.

Vale destacar que la magnitud del efecto expuesto podría estar subestimada, ya que no se está controlando por el hecho de haber tenido un hijo durante el pregrado. Resulta lógico pensar que, producto del embarazo, el efecto de tener un hijo tiene mayor impacto en el exceso de duración incurrido en el caso de las mujeres que en el de los hombres. Por ende, si se estuviera controlando por el hecho de haber vivido un embarazo, probablemente la diferencia promedio de sobreduración entre mujeres y hombres, de iguales características, sería más pronunciada que la presentada.

\subsection{Relevancia de los antecedentes de la enseñanza media}

Adicionalmente, el estimador obtenido para el indicador de NEM se condice con la lógica esperada: dos estudiantes provenientes

16 Dado que la sobreduración promedio en carreras universitarias es de 3 semestres, un $1,2 \%$ de este periodo de 78 semanas equivale a 0,94 semanas. De esta forma se puede convertir el exceso porcentual de sobreduración obtenido para cada estimador, para lo cual se debe tener en cuenta que el exceso de duración promedio en CFT es 2 semestres y en los IP es de 1,7 semestres. 
de colegios similares, con contextos socioeconómicos parecidos, que cursan la misma carrera en la misma IES pero que tuvieron rendimientos académicos dispares durante su paso por la enseñanza media, incurren en niveles de sobreduración distintos. Como se planteó, un egresado con mejores calificaciones probablemente estará mejor preparado para afrontar las exigencias impuestas durante la ES, lo cual se traduciría en menores niveles de exceso de duración en promedio. Los coeficientes estimados sugieren que, dependiendo del tipo de IES, por cada decil NEM de diferencia se genera una disminución de entre 1 y 1,5 puntos porcentuales en la TSD.

Un estudiante universitario que egresó de un colegio municipal sobredura en promedio 3,17 puntos porcentuales más (2,5 semanas más) que un par de iguales características proveniente de un colegio particular pagado. En el caso de los CFT e IP, dicha brecha se reduce a 2 y 0,5 semanas, respectivamente.

\subsection{Incentivos generados por los mecanismos de financiamiento}

Respecto de los resultados expuestos para el modelo $\mathrm{N}^{\circ} 2$, nuevamente se obtiene que todos poseen una alta significancia estadística. Los coeficientes reportados para las variables binarias de gratuidad y becas se alinean con la evidencia internacional: aquellos estudiantes que asumirían una carga financiera relativamente mayor producto de su atraso, tienden a esforzarse más para terminar sus estudios a tiempo. En concreto, la sobreduración reportada para individuos similares cae sustancialmente si el estudiante fue beneficiario de la política de gratuidad.

La intuición detrás del resultado es simple: un individuo que goza de gratuidad tiene un incentivo financiero para terminar a tiempo, ya que, si no lo hace, tendrá que comenzar a pagar por sus estudios. De esta forma, alumnos de características observables idénticas, que antes estudiaban las mismas carreras sin gratuidad, hoy incurren en un exceso de duración promedio mucho menor: 13, 5 y 7 puntos porcentuales menos en CFT, IP y universidades, respectivamente. Esto equivale a una reducción del 23\% en la TSD 
—o bien de un mes y medio del exceso de duración- promedio reportada por estudiantes universitarios provenientes de sectores vulnerables que antes estudiaban sin gratuidad, ceteris paribus. En el caso de los estudiantes que asisten a CFT, la TSD se redujo en un 30\% solo por ser beneficiario de la gratuidad. La evidencia es contundente y está alineada con los resultados obtenidos por Gunnes et al. (2013), en los que se demuestra que los estudiantes tienen presupuestos acotados e incorporan rápidamente el impacto que tiene un mayor esfuerzo sobre el tiempo que demoran en culminar sus carreras.

Producto de que los incentivos a terminar a tiempo que impone la gratuidad también son válidos para las IES, se podría argumentar que estas tendrían incentivos a exigirle mayor celeridad y carga académica a los estudiantes que son beneficiarios de la política, para minimizar la cantidad de beneficiarios que exceden la duración nominal de la carrera. Esta presión por parte de las IES podría provocar un aumento de los niveles de deserción de los estudiantes vulnerables, lo cual, a su vez, generaría que el subgrupo de estudiantes vulnerables para los cuales se puede estudiar la tasa de sobreduración sea más pequeño: aquellos que soportaron la presión académica por terminar a tiempo.

Si este fuese el caso, resulta plausible asumir que, luego de la implementación de la gratuidad, los estudiantes vulnerables que logran terminar sus carreras corresponden a una muestra más hábil y preparada, por lo que la reducción de la TSD obtenida para los beneficiarios de la gratuidad estaría condicionada por el sesgo de selección mencionado. Para descartar o corroborar la hipótesis planteada se calcularon las cifras de deserción al primer, segundo y tercer año de los estudiantes vulnerables por cohorte de ingreso a la ES.

Como se puede apreciar en el gráfico $\mathrm{N}^{\circ} 1$, en lugar de observarse un aumento de la deserción promedio de los estudiantes vulnerables luego de la implementación de la gratuidad, se constata una reducción sostenida de los niveles de deserción al primer, segundo y tercer año para este grupo de estudiantes. Esto permitiría descartar la hipótesis de que el estimador del efecto de la gratuidad 
sobre la TSD podría estar sobrestimado por el sesgo de selección mencionado.

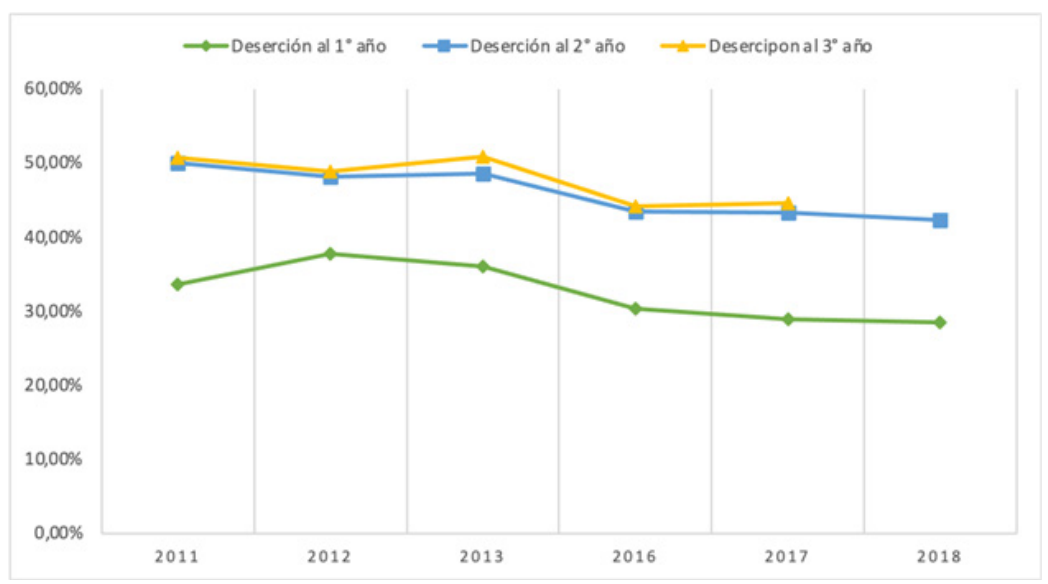

Gráfico $\mathrm{N}^{\circ} 1$. Evolución de la deserción promedio de los estudiantes de los seis primeros deciles, por cohorte de ingreso a la Educación Superior.

Fuente: elaboración propia basada en datos del Mineduc.

En el caso de los becados la interpretación del coeficiente resultante es similar: quienes estuvieron becados durante su paso por la educación terciaria tienden a reportar tasas de sobreduración entre 4 y 6 puntos porcentuales más bajas que sus compañeros de características similares que no gozaron de ningún tipo de beca. Parte del efecto también puede explicarse por los incentivos que tiene el individuo a no perder la beca, los cuales hacen que este se esfuerce más por cumplir las exigencias académicas y, en consecuencia, termine sus estudios más temprano.

No obstante, se debe tener en consideración que el ponderador podría estar sobrestimado, ya que los individuos becados podrían haber sido seleccionados por sus conocimientos. Estas características a veces son difíciles de cuantificar y podrían no haber sido capturadas por las otras variables explicativas del modelo. Por tanto, dada la alta correlación que pueden tener las habilidades de los alumnos con el hecho de haber sido becados, el ponderador de beca estaría incorporando una disminución adicional de la sobreduración generada por ser más hábil. 
En el caso de los créditos de ES, el efecto depende del tipo de IES. En los CFT, haber sido usuario de un crédito se asocia a niveles de sobreduración más bajos, mientras que en las universidades y en los IP se observa que los beneficiarios de los créditos reportan una TSD más alta que sus pares de características observables similares. Una posible explicación del fenómeno anterior radica en el hecho de que la gran mayoría de los beneficiarios de los créditos debe copagar el arancel por sus propios medios, ya que no es posible solicitar un crédito por la totalidad del arancel cobrado. Por ende, muchos de ellos trabajan mientras estudian, para financiar la diferencia no cubierta por el crédito.

Como demostraron Stinebrickner \& Stinebrickner (2003), cuando un individuo trabaja y estudia simultáneamente tiende a destinar menos tiempo al cumplimiento de sus objetivos académicos, lo cual genera un enlentecimiento en la obtención de su título. Parece lógico que dentro del grupo de individuos que estudió con crédito se observe una mayor proporción que trabajó mientras cursaba su carrera; resulta esperable que la variable de crédito esté capturando el efecto mencionado.

\section{4 ¿Estudiantes internalizan el costo de oportunidad?}

Como se puede apreciar en la tabla $\mathrm{N}^{\circ} 9$, el valor del arancel tiene un mayor impacto en la TSD incurrida por estudiantes del sector técnico profesional: ante un aumento del 30\% en el arancel cobrado, se reporta una caída de 10 y 8 puntos porcentuales en la TSD percibida por los estudiantes matriculados en CFT e IP, respectivamente. En cambio, en el caso de los estudiantes universitarios dicha brecha se reduce a la mitad.

\subsection{Impacto atenuado de la vulnerabilidad}

Los estimadores del primer modelo sugieren que un individuo vulnerable demoraría menos en cursar la misma carrera que un compañero proveniente de sectores más acomodados. Considerando las ventajas que tiene un individuo que proviene de una familia con una mejor situación socioeconómica, sería lógico que este tenga 
más facilidades para culminar sus estudios a tiempo. Al analizar los resultados del modelo $\mathrm{N}^{\circ} 2$ se puede inferir que el estimador de vulnerabilidad del primer modelo habría estado capturando el efecto de otras variables que son más recurrentes en el grupo alumnos vulnerables y que, a su vez, impactan de manera negativa la sobreduración incurrida por los estudiantes: becas y gratuidad.

En efecto, al incorporar la utilización de los distintos canales de financiamiento al modelo, el impacto reportado para la vulnerabilidad cambió. Se demostró que quienes fueron usuarios de la gratuidad o fueron becados incurrieron en un exceso de duración mucho menor. Además, los individuos vulnerables son los únicos que pueden haber sido beneficiarios de la gratuidad y son quienes reciben la mayoría de las becas. Por ende, dada la alta correlación existente entre la vulnerabilidad y las variables de beneficios financieros, el hecho de omitir estas últimas en el primer modelo generaba que su efecto fuera capturado en parte por el indicador de vulnerabilidad, provocando un sesgo a la baja en el coeficiente obtenido.

En pocas palabras, el hecho de ser vulnerable no implica un menor nivel de sobreduración como se infería del primer modelo. De hecho, el segundo modelo sugiere que los individuos vulnerables perciben una TSD promedio levemente más elevada que sus compañeros provenientes de sectores más adinerados, de idénticas características observables. Sin embargo, la magnitud del efecto encontrado en el segundo modelo sigue siendo menor: entre 0,4 y 0,7 puntos porcentuales de diferencia en la TSD, dependiendo del tipo de IES.

Diversos estudios han diagnosticado que la vulnerabilidad debe ser entendida de forma multidimensional y no solo desde una perspectiva económica al momento de analizar su impacto sobre las trayectorias educativas (Carrillo Álvarez, Civís Zaragoza, Andrés Blanch, Longás Mayayo, E. y Riera Romaní, 2018; DíazBarriga, Vásquez-Negrete y Díaz David, 2019). Señalan que existen factores socioemocionales, individuales, nutricionales, familiares y/o comunitarios que podrían generar efectos perjudiciales sobre el éxito académico en todos los niveles educativos. Para intentar incorporar 
una medición de la vulnerabilidad multidimensional al estudio se incorporó en el tercer modelo el IVE (índice de vulnerabilidad escolar, basado en la interacción de factores de riesgo sociales, económicos, psicológicos, culturales y/o ambientales).

Los resultados del tercer modelo se alinean con la evidencia mencionada, ya que se infiere que el efecto de la vulnerabilidad multidimensional sobre la TSD es de una magnitud mayor que el obtenido al considerar como único proxy de vulnerabilidad el ingreso socioeconómico. En efecto, un estudiante universitario que egresó de un establecimiento municipal ubicado en una comuna con un bajo IVE, como Vitacura, incurre en una TSD 2,3 puntos porcentuales más baja que un par de iguales características, con una trayectoria académica similar, que egresó de un colegio ubicado en una comuna con un IVE elevado, como La Pintana.

\section{Conclusiones}

En suma, a lo largo del estudio se realizó un análisis multivariado de los potenciales determinantes de la sobreduración en la ES. Realizando un análisis censal, se identificaron varios patrones al interior de la ES chilena que se alinean con la evidencia recopilada al respecto en el ámbito internacional. Además, los resultados entregan una primera aproximación acerca de cómo se podría eventualmente mitigar el exceso de duración percibido por los estudiantes chilenos.

En primer lugar, se identificó una brecha de género estadísticamente significativa, que sugiere que las mujeres demoran menos que los hombres en cursar el pregrado.

Por otro lado, se demostró que la política de gratuidad en la ES puso incentivos sobre los estudiantes vulnerables a terminar sus carreras más rápido. De hecho, la TSD reportada cambia radicalmente para individuos similares solo por el hecho de haber financiado sus estudios a través de la gratuidad: estudiantes de características idénticas antes de la implementación de la gratuidad sobreduraban casi un 30\% más. 
Por ende, si se extendiera la cobertura de la gratuidad a los años de sobreduración, se podría presumir que el incentivo mencionado desaparecería y estos alumnos volverían a percibir los niveles de sobreduración reportados antes de la implementación de esta política. Esto sería un efecto indeseado, ya que incrementaría el gasto público generado por la gratuidad, toda vez que se estaría perjudicando precisamente a quienes se pretende beneficiar.

En la misma línea, los individuos que fueron becados durante su paso por la educación terciaria tienden a incurrir en excesos de duración menores que sus compañeros de características similares que no gozaron de ningún tipo de beca, ya que poseen un incentivo al esfuerzo que radica en la posibilidad de perder la beca si no cumplen con ciertos estándares académicos. A grosso modo, se evidenció que los incentivos a terminar a tiempo que impone la posibilidad de perder un beneficio de financiamiento hacen que estudiantes de igual características sobreduren menos.

Contrariamente, un estudiante universitario que financió su carrera gracias a las ayudas crediticias del Estado demora un poco más que un par, con antecedentes parecidos, que no solicitó dicho beneficio. Una hipótesis que podría explicar dicha diferencia es que la amplia mayoría de los usuarios de los créditos debe copagar parte del arancel por sus propios medios, razón por la cual muchos de ellos se ven obligados a trabajar mientras estudian para financiar el saldo restante. La evidencia internacional encontrada sugiere que quienes estudian y trabajan simultáneamente demoran más en concluir sus procesos educativos terciarios. La reducción de los niveles de copago podría presentarse como una eventual solución al problema anterior, para así permitir que los estudiantes puedan concentrar todos sus esfuerzos en cumplir las exigencias académicas.

Adicionalmente, los resultados permitieron confirmar la hipótesis de que los individuos que tuvieron un mejor rendimiento académico, en comparación con sus pares de enseñanza media, tienden a percibir excesos de duración más atenuados. No obstante, a pesar de controlar por todos los factores observables estudiados, quienes egresan de colegios municipales tienen una TSD basal más 
elevada. Consecuentemente, los egresados de colegios particulares pagados sobreduran mucho menos que sus pares, a pesar de presentar antecedentes similares. El resultado anterior no es más que un reflejo de las brechas de calidad existentes en el sistema educativo chileno, cuya única solución posible es el fortalecimiento de la educación pública en todos sus niveles.

Por último, vale destacar que el presente estudio busca realizar una primera aproximación al análisis de los determinantes del exceso de duración en la educación terciaria chilena y, en ningún caso, determinar la cuantía exacta de un vínculo causal. De esta forma, la evidencia expuesta permite sentar las bases teóricas y empíricas para futuros estudios en la materia, que faciliten la construcción de evidencia más robusta, de cara a la elaboración e implementación de políticas públicas que permitan disminuir los niveles de sobreduración incurridos por los estudiantes chilenos.

\section{Referencias}

Adelman, C. (2006). The Toolbox Revisited: Paths to Degree Completion from High School Through College. U.S. Department of Education. Retrieved from: www.ed.gov/rschstat/research/pubs/toolboxrevisit/ index.html

Allen, J. \& Robbins, S. (2010). Effects of interest-major congruence, motivation, and academic performance on timely degree attainment. Journal of Counseling Psychology, 57(1), 23-35. DOI: https://doi. org/10.1037/a0017267

Bettinger, E. \& Long, B. T. (2009). Addressing the Needs of Underprepared Students in Higher Education. Journal of Human Resources, 44(3), 736-771. Retrieved from: http://jhr.uwpress.org/content/44/3/736. abstract

Betts, J. (1996). What Do Students Know about Wages? Evidence from a Survey of Undergraduates. The Journal of Human Resources, 31(1), 27-56. Retrieved from: https://www.jstor.org/stable/146042

Bucarey, A., Contreras Guajardo, D. \& Muñoz, P. (2020). Labor Market Returns to Student Loans for University: Evidence from Chile. Journal of Labor Economics, 38(3). DOI: https://doi.org/10.1086/706486

Carrillo Álvarez, E., Civís Zaragoza, M., Andrés Blanch, T., Longás Mayayo, E. y Riera Romaní, J. (2018). Condicionantes del éxito y fracaso escolar 
en contextos de bajo nivel socioeconómico. Revista de Estudios y Experiencias en Educación, 2(1), 75-94. Recuperado de: http://www. rexe.cl/ojournal/index.php/rexe/article/view/492

Carvajal, C., Gonzalez, J. A. Tassara, C. A. y Álvarez, M. S. (2018). Sobre-duración: una Aproximación Cuantitativa. Formación Universitaria, 11(3), 19-28. DOI: http://dx.doi.org/10.4067/S071850062018000300019

Deming, D. J., Hastings, J. S., Kane, T. J. \& Staiger, D. O. (2014). School Choice, School Quality, and Postsecondary Attainment. American Economic Review, 104(3), 991-1013. DOI: https://doi.org/10.1257/ aer.104.3.991

DesJardins, S. L., Ahlburg, D. A. \& McCall, B. P. (2002). A Temporal Investigation of Factors Related to Timely Degree Completion. The Journal of Higher Education, 73(5), 555-581. DOI: http://doi. org/10.1353/jhe.2002.0042

Díaz-Barriga, F., Vásquez-Negrete, V. I. y Díaz David, A. (2019). Sentido de la experiencia escolar en estudiantes de secundaria en situación de vulnerabilidad. Revista Latinoamericana de Ciencias Sociales, Niñez y Juventud, 17(1), 237-252. DOI: https://doi.org/https://dx.doi. org/10.11600/1692715x.17114

Garibaldi, P., Giavazzi, F., Ichino, A. \& Rettore, E. (2012). College Cost and Time to Complete a Degree: Evidence from Tuition Discontinuities. Review of Economics and Statistics, 94(3), 699-711. DOI: https://doi. org/10.1162/REST_a_00195

Goodman, J., Hurwitz, M. \& Smith, J. (2017). Access to 4-Year Public Colleges and Degree Completion. Journal of Labor Economics, 35(3), 829-867. DOI: https://doi.org/10.1086/690818

Gunnes, T., Kirkebøen, L. J. \& Rønning, M. (2013). Financial incentives and study duration in higher education. Labour Economics, (25), 1-11. DOI: https://doi.org/10.1016/j.labeco.2013.04.010

Hämäläinen, U., Koerselman, K. \& Uusitalo, R. (2017). Graduation Incentives Through Conditional Student Loan Forgiveness. Discussion Papers 11142, Institute of Labor Economics (IZA). Retrieved from: http://ftp. iza.org/dp11142.pdf

Herzog, S. (2005). Measuring determinants of student return vs. dropout/ stopout vs. transfer: A first-to-second year analysis of new freshmen. Research in Higher Education, 46(8), 883-928. DOI: https://doi. org/10.1007/s11162-005-6933-7 
Hill, J., Smith, N., Wilson, D. \& Wine, J. (2016). 2012/14 Beginning Postsecondary Students Longitudinal Study (BPS:12/14): Data File Documentation (NCES 2016-062). U. S. Department of Education. Washington, DC: National Center for Education Statistics. Retrieved from: https://nces.ed.gov/pubs2016/2016062.pdf

Montoya, A. M., Noton, C. \& Solis, A. (2018). The Returns to College Choice: Loans, Scholarships and Labor Outcomes. Working Paper Series, (12), Uppsala University, Department of Economics. Retrieved from: https:// ideas.repec.org/p/hhs/uunewp/2018_012.html

OECD. (2019). Education at a Glance 2019: OECD Indicators. Paris: OECD Publishing. DOI: https://doi.org/10.1787/f8d7880d-en.

Oriol Granado, K., Mendoza Lira, M., Covarrubias Apablaza, A. G. y Molina López, V. M. (2017). Emociones positivas, apoyo a la autonomía y rendimiento de estudiantes universitarios: El papel mediador del compromiso académico y la autoeficacia. Revista Psicodidáctica, 22(1), 45-53. DOI: https://doi.org/10.1387/RevPsicodidact.14280

Pey, R., Durán, F. y Jorquera, P. (2012). Informe para la toma de decisiones sobre duración de las carreras de pregrado en el CRUCH. Consejo de Rectores de las Universidades Chilenas (CRUCH). Recuperado de: http://reforma.fen.uchile.cl/Papers/DURACION\%20CARRERAS\%20 PREGRADO\%20CRUCH.pdf

Scott-Clayton, J. E. (2015). The Role of Financial Aid in Promoting College Access and Success: Research Evidence and Proposals for Reform. Journal of Student Financial Aid, 45(3). Retrieved from: https://ir.library. louisville.edu/jsfa/vol45/iss3/3

Stinebrickner, R. \& Stinebrickner, T. R. (2003). Working during School and Academic Performance. Journal of Labor Economics, 21(2), 473-491. Retrieved from: https://www.jstor.org/stable/10.1086/345565

Sten-Gahmberg, S. (2020). Student Heterogeneity and Financial Incentives in Graduate Education: Evidence from a Student Aid Reform. Education Finance and Policy, 15(3), 543-580. Retrieved from: https://doi. org/10.1162/edfp_a_00294

Valenzuela, J. P. Allende González, C., Gómez Vera. G. y Trivelli, C. (2015). El efecto de la SEP en la reducción de la segregación socioeconómica del sistema escolar chileno. Primeros resultados. Estudios de Política Educativa - Mineduc, (1), 14-61. Recuperado de: https://www. researchgate.net/publication/280557341

Vera-Noriega, J. A., Ramos-Estrada, D. Y., Sotelo-Castillo, M. A., EcheverríaCastro, S., Serrano-Encinas, D. M. y Vales-García, J. J. (2012). 
244 DETERMINANTES DEL EXCESO DE DURACIÓN EN LA EDUCACIÓN TERCIARIA EN CHILE - S. Carpentier

Factores asociados al rezago en estudiantes de una institución de educación superior en México. Revista Iberoamericana de Educación Superior, 3(7), 41-56. Recuperado de: https://www.redalyc.org/ pdf/2991/299129031003.pdf

Vossensteyn, J. J., Kottmann, A., Jongbloed, B. W. A., Kaiser, F., Cremonini, L., Stensaker. B., Hovdhaugen, E. \& Wollscheid, S. (2015). Dropout and Completion in Higher education in Europe. Publications Office of the European Union, Luxembourg. DOI: http://dx.doi. org/10.2766/826962.

Recibido: 27/01/2021

Aceptado: 24/05/2021 